E.L.U.A., 6, 1990, pp: 229-239.

\title{
COHERENCIA TEXTUAL: ¿COHERENCIA INTERNA O COHERENCIA EXTERNA?
}

\author{
ANTONIO VILARNOVO \\ (Universidad de Navarra)
}

0.1. Sobre coherencia textual se ha escrito mucho en los últimos años. No es nuestro propósito realizar aquí un estado de la cuestión minucioso sobre las distintas teorías explicativas de la coherencia textual, cosa que ya hemos hecho en un artículo de próxima publicación, titulado «Teorías explicativas de la coherencia textual». Nuestra intención es mostrar cómo los distintos tratamientos del problema han tomado dos direcciones fundamentales: o bien han intentado explicar la coherencia por un principio interno al texto (coherencia interna), o bien han buscado dar cuenta de la coherencia por un principio exterior al texto (coherencia externa). También mostraremos cómo sólo se puede dar una explicación satisfactoria de la coherencia partiendo del texto mismo, e indicaremos en qué medida colaboran los factores externos —en especial la designación y el conocimiento del mundo- al establecimiento de la coherencia textual.

0.2. Antes de pasar a la exposición de las distintas teorías sobre la coherencia de los textos, digamos algo sobre el concepto mismo de coherencia textual.

\section{DEFINICIÓN DE COHERENCIA}

1.1. Para M. E. Conte (1988b) el término coherencia tiene dos acepciones. En una primera, coherencia se define negativamente, como no-contradictoriedad: ausencia de contradicción; en este sentido, un texto coherente sería, entre otras cosas, un texto sin contradicciones. En la segunda acepción, se significa la conexión de las partes en un todo: la interrelación de los distintos elementos del texto. En opinión de la autora, la primera propiedad, la no-contradictoriedad, no sería una propiedad necesaria de los textos, sino una cualidad contingente; 
así, según M. E. Conte (1988b), habría textos que contienen contradicciones entre sus enunciados, e incluso habría textos formados por un único enunciado contradictorio. En cambio, la segunda propiedad, la conexión de las partes en un todo, correspondería a la quidditas misma del texto, y sí sería esencial para la coherencia.

1.2. Haremos ahora unas observaciones a estas tesis sumamente interesantes de Conte. En nuestra opinión, la segunda propiedad señalada por la autora, la conexión de las partes (cohesión) habría que incluirla en una propiedad más general: la de la unidad de todo texto. Desde este punto de vista, la cohesión sería la manifestación linguística de la unidad de los textos, que es sobre todo -aunque no sólo- una unidad de sentido (utilizaremos aquí el término sentido, con el valor que le da Coseriu, 1981a). Por lo que respecta a la primera propiedad, a la no-contradicción, pensamos que ésta es también esencial para la coherencia de los textos, pues la contradicción, a nuestro parecer, sólo sería aceptable en dos casos: a) cuando es explicable por alguna razón, con lo cual desaparece la incoherencia; b) cuando la contradicción contribuye al sentido del texto, con lo que vuelve a desaparecer la incoherencia (piénsese, p.ej., en el llamado «teatro del absurdo»). Además, pensamos que esta propiedad podría incluirse en la segunda nota (la de la unidad), en la medida en que la contradicción supone una cierta ruptura de esta característica, al atentar contra la unidad de sentido.

1.3. Una vez visto esto, pasemos a la revisión de las distintas teorías sobre la coherencia textual.

\section{EXPLICACIÓN POR LA COHESIÓN}

2.1. Muy al comienzo de los estudios sobre la coherencia ésta se ha intentado explicar a través de la cohesión, es decir: por la interrelación linguíística de las distintas partes de un texto. Según esto, el texto sería como un tejido donde unas partes remiten a otras. Entre los autores que han seguido esta línea explicativa destacan: Pêcheux (1969), I. Bellert (1970), Charolles (1978), Lundquist (1980), Giora (1985), F. Fillol y J. Mouchon (1978), T. Reinhart (1980), A. Lecomte y J. Marandin (1986), B. Combettes (1986).

La cohesión, según Lundquist (1980), se conseguiría por tres tipos de procedimientos: 1) la correferencia : la utilización de varias instancias lingüísticas que designan un mismo objeto extralingüístico; 2) la contigüidad semántica: es decir, la utilización recurrente de elementos semánticos próximos; 3) los conectores: el uso de partículas que sirven de ligazón entre las distintas partes del discurso.

2.2. Dentro de esta línea hay que situar el artículo de Bellert (1970). Según esta autora, la cohesión sería una condición necesaria, aunque no suficiente, de la coherencia textual. Es decir, Bellert (1970) entiende que la interrelación de 
las partes del discurso no constituye propiamente la coherencia, pero que sí sería una condición necesaria en todo texto coherente. En nuestra opinión, la cohesión tampoco sería una condición necesaria para la coherencia. En el artículo anteriormente citado hemos mostrado cómo pueden construirse textos muy breves perfectamente coherentes y que sin embargo no tienen cohesión. En realidad caben con respecto a esto las cuatro posibilidades teóricas: textos coherentes con cohesión, coherentes sin cohesión, no coherentes con cohesión y no coherentes sin cohesión. Mostraremos a continuación con ejemplos cómo puede darse la segunda posibilidad señalada:
(1)
(a) Me voy al campo.
(b) Adiós.
(a) La luna es redonda.
(b) Los hechos son incuestionables.

En (1) y (2) la relación entre las secuencias no se establece mediante mecanismos lingüísticos de cohesión; la relación la ponemos nosotros por nuestro conocimiento del mundo. Así, en (1) sabemos que cuando alguien se va se le suele decir adiós o cualquier otra fórmula de cortesía, y por eso percibimos el tex to como coherente. En (2) sucede algo parecido: es nuestro conocimiento del mundo el que nos permite interpretar (2) (a) como un hecho. Por lo tanto, puede haber textos coherentes sin cohesión: la cohesión no es una propiedad necesaria para la coherencia.

2.3. No obstante, lo verdaderamente importante de este intento de explicación es que supone un deseo de dar cuenta de la coherencia a través del texto mismo (coherencia interna).

\section{EXPLICACIÓN POR EL TÓPICO DEL DISCURSO}

3.1. La segunda teoría sobre la coherencia textual es la que propone una explicación a través del tema o tópico del discurso. Esta línea de investigación es la que ha dado los mejores resultados hasta el momento. Se trata también de un intento de justificar la coherencia por medio del texto mismo. Entre los autores que han seguido esta dirección hay que situar a: Charolles (1978), Reinhart (1980), Laparra (1982), Hobbs (1983), Blum-Kulka (1983), Giora (1985), Abadi (1986), Lundquist (1986), Combettes (1986), etc.

3.2. Cuando se trabaja con la bibliografía relativa al tema o tópico del discurso sorprende el hecho de que se entiende por tema o tópico del discurso lo que en la bibliografía sobre tema y rema se entiende por rema. Esto ha llevado a algunos autores (Bernárdez,1982) a decir que se trata de cosas distintas, que no deben confundirse. A nuestro parecer no se trata propiamente de cosas distintas, aunque sí sea necesario distinguir. Se trata en realidad del mismo 
concepto de tema, aunque referido a niveles de aplicación distintos. Se hace, pues, necesario distinguir, con vistas a nuestra explicación, entre tema y rema en la frase, en el discurso y en un conjunto de discursos (Coseriu, 1981 a), pues tema y rema no funcionan de la misma manera en estos tres niveles. La distinción que nos interesa aquí es la de tema y sema en el discurso. Decíamos anteriormente que podría buscarse un concepto unitario de tema (y rema), y que únicamente habría variaciones dependiendo del nivel de aplicación. Básicamente podría definirse tema/rema como given/new (lo dado, conocido/lo desconocido o añadido), lo que en el discurso sería: el asunto sobre el que se habla/lo que se añade de nuevo. Ahora bien, llegados a este punto es necesario distinguir, una vez más, dentro de tema y rema en el discurso: a) en la constitución del discurso; b) en el discurso ya constituído. En la constitución del discurso, ha mostrado Danes (1970), y otros, que tema y rema se articulan en cada una de las secuencias o frases del texto, de forma que se va añadiendo progresivamente la información; y esto de diferentes modos (Danes, 1970). De forma que al final se tiene un texto completo. Pues bien, en el discurso ya constituido, lo que se obtiene es un único tema y rema, pero de forma que tema y rema coinciden, porque el asunto del texto (tema) es justamente lo que dice o añade (rema). Por tanto, no hay ningún problema para que se entienda por tópico del discurso el rema, porque en el discurso ya constituído tema y rema coinciden.

3.3. Tras este breve excurso, retornamos a la explicación de la coherencia por el tema o tópico del discurso. Tal vez haya sido Giora (1985) quien haya aportado más en esta dirección. Según Giora (1985), para que un texto sea coherente debe cumplir dos condiciones:

1) Que cada una de las secuencias del discurso sea interpretable como perteneciente al mismo tema.

2) Que las digresiones sean señaladas como digresiones por una marca distintiva de digresión.

Así, el texto

(3) Juan es amigo de Fernando.

Fernando vino a casa ayer

es coherente por cumplir la primera condición, ya que las dos secuencias hablan sobre un mismo tema: Fernando. Si añadimos una nueva secuencia:

(4) Juan es amigo de Fernando

Fernando vino a casa ayer

Por cierto ayer fue un día lluvioso,

el texto sigue siendo coherente, pues la digresión queda señalada como digresión con el marcador por cierto. 
3.4. Sin embargo, no es suficiente con estas condiciones para dar cuenta de la coherencia mediante el tópico del discurso. Es necesario añadir, al menos tres nuevos requisitos:

1) La no-contradicción.

2) La ausencia de saltos lógicos entre las secuencias.

3) La ordenación por subtemas.

Ya hemos visto que la contradicción va en contra de la coherencia por romper la unidad de sentido. Así sucede en el texto

\section{(5) Los cuatro puntos cardinales son tres: norte y sur,}

porque cuatro nunca puede ser igual a tres, ni tres igual a dos. Igualmente, tendemos a rechazar los textos con saltos lógicos, por faltos de unidad, y los textos desordenados, porque cuesta percibir la unidad temática.

3.5. En conclusión: mediante el concepto de tema o tópico del discurso pueden darse explicaciones válidas sobre la coherencia textual. La investigación lingüística deberá seguir avanzando en esta dirección, pero es éste indudablemente un buen camino. Lo verdaderamente importante aquí es que se trata de una teoría que parte del texto mismo, que explica la coherencia por un principio interno al texto, y no por un factor exterior al propio texto.

3.6. Hasta este punto hemos visto las teorías que dan cuenta de la coherencia por un procedimiento de «coherencia interna». Ahora veremos otros dos tratamientos de la cuestión que se basan en un principio exterior al texto.

\section{COHERENCIA COMO INTERPRETACIÓN}

4.1. Dentro de esta tendencia hay que situar a Sgall (1983), Charolles (1983 y 1985), Neubauer (ed.) (1983), Hatakeyama, Petöfi y Sözer (1985), Conte (1988c). Según este intento explicativo, la coherencia no estaría tanto en el texto como en el sujeto intérprete del texto; la coherencia sería más bien una propiedad atribuida por el intérprete y no algo objetivo de los textos. Naturalmente, aquí se sitúa la coherencia en un elemento externo al texto: en este caso, en el emisor o receptor, que sería quien dotaría de sentido al discurso.

4.2. Sin embargo, no todos los autores que sitúan la coherencia en el intérprete niegan una propiedad objetiva de los textos. Así, Conte (1988c) distingue una coherencia a parte subjecti (o por parte del sujeto) y una coherencia a parte objecti (por parte del objeto). Es decir, reconoce una propiedad objetiva de los textos por la cual éstos serían coherentes, y, juntamente con ella, una tendencia en el intérprete a atribuir coherencia a los textos. Con relación a esto, el interpretar sería un proceso dialéctico entre el sujeto y el objeto. Desde este 
punto de vista, el texto podría entenderse como una secuencia de instrucciones que guían al receptor en la interpretación del sentido y en la construcción de la coherencia.

4.3. Nos parece oportuno señalar aquí la necesidad de relacionar los dos tipos de coherencia (por parte del sujeto y por parte del objeto), en el sentido siguiente: la coherencia por parte del objeto es el fundamento de la coherencia por parte del sujeto, en la medida en que el sujeto es capaz de atribuir coherencia a los textos cuando ellos ya tienen algo objetivo que permite esta atribución: toda interpretación se realiza con base en el texto mismo, y éste es siempre de un modo determinado: nadie ha confundido jamás El Quijote con La Celestina. Por otra parte, y de otro modo, la coherencia por parte del sujeto va más allá de la coherencia por parte del objeto: la interpretación es un proceso activo en el que el intérprete pone algo. Interpretar no es simplemente descodificar; es una actividad constructiva en la que se añaden cosas que no están en el texto, en particular: 1) el conocimiento del mundo; 2) los principios generales del pensamiento; 3) la reconstrucción de los contextos (Coseriu, 1981). En este sentido indica muy certeramente Conte (1988c:91) que interpretar es una actividad muy semejante a la de construir un puzzle: en los dos casos se trata de formar una imagen coherente, pero en la interpretación algunas piezas tenemos que hacerlas nosotros. No obstante, también en este caso, el sentido del texto y lo que el texto mismo permite es el criterio de la interpretación y, por lo tanto, de la coherencia por parte del sujeto.

4.4. Lo verdaderamente importante de esta postura es que se trata de un intento de explicar la coherencia por un elemento exterior al texto mismo: en este caso, por el sujeto intérprete. Hemos visto cómo es necesario hacer compatibles los dos tipos de de coherencia y cómo, en última instancia, la coherencia por parte del sujeto depende también del texto (en el sentido indicado en 4.3.).

\section{COHERENCIA COMO CONEXIÓN ENTRE ESTADOS DE COSAS}

5.1. Finalmente, nos referimos ahora, y de un modo más extenso, a una cuarta teoría, que sitúa el criterio de la coherencia en las cosas mismas: se trata de entender la coherencia como «conexión entre estados de cosas». Dentro de esta línea hay que situar preferentemente a Van Dijk (1983). Para Van Dijk (1983), un texto es coherente cuando se pueden establecer relaciones entre las distintas proposiciones o secuencias por la relación entre los estados de cosas designados. Según esto lo decisivo para la conexión entre las secuencias es la conexión entre las cosas; por lo tanto, la realidad exterior se convertiría en la norma de la coherencia textual, en lugar de situar las condiciones de coherencia en el propio texto. 
5.2. Van Dijk (1983) distingue cinco modos de estar relacionadas las circunstancias externas, y por lo tanto las proposiciones:

(a) A es causa de B.

(b) A es una fundamentación de B (siendo B una actuación).

(c) A y B ocurren en la misma situación, en el mismo par mundo-tiempo, y pertenecen al mismo ámbito conceptual.

(d) A es lógicamente o conceptualmente parte de B.

(e) A es una «parte integrante» convencional de B.

Estos son los cinco modos en que pueden relacionarse las circunstancias externas y las proposiciones. Por lo tanto, un texto será coherente si se pueden descubrir este tipo de vínculos entre secuencias.

5.3. Además, Van Dijk (1983) indica que esto sería válido para cualquier mundo posible, con lo que extiende su aplicación a la literatura y a los mundos que ésta crea.

5.4. Hay una serie de similitudes y diferencias entre este modo de entender la coherencia y el del tema o tópico del discurso:

a) En ambas teorías se mantiene la necesidad de una ligazón entre las proposiciones o secuencias: en este caso la conexión se establece por la relación entre los referentes, mientras que en el caso de la explicación por el tema del discurso ésta se establece por la ordenación de las secuencias en torno a un mismo tema.

b) En los dós tipos de explicación se defiende la importancia del conocimiento del mundo como elemento fundamental para atribuir coherencia a los textos: en la teoría del tópico del discurso se trata de un uso instrumental de ese conocimiento, mientras que en esta otra teoría el conocimiento del mundo sería el criterio y fundamento mismo de la coherencia.

c) La diferencia más radical entre las dos teorías estriba en el lugar donde se sitúa el criterio de coherencia: en un principio interno al texto (tópico del discurso), o externo (conexión entre estados de las cosas).

5.5. Pues bien, he aquí la doble posibilidad: coherencia interna o coherencia externa. En nuestra opinión es preciso optar por una explicación de la coherencia que se centre en el texto mismo, ya que se trata precisamente de explicar la coherencia textual. A nuestro parecer, la relación entre los estados de cosas no puede ser criterio definitivo para atribuir coherencia a un texto, y esto por tres razones:

1) La cantidad de relaciones posibles entre los objetos externos son potencialmente infinitas. De manera que, según esto, todos los textos serían coherentes, porque siempre sería posible encontrar una relación entre los objetos; sin embargo, lo que se comprueba es que hay textos que no son coherentes. 
2) Se comprueba que textos con los referentes relacionados entre sí pueden carecer de toda lógica y coherencia, por carecer de un tema unitario o por presentarse desordenadas sus secuencias, o por otros motivos.

3) Finalmente, y esto es lo más importante, lo que se comprueba en los textos (sobre todo en los literarios, pero no sólo en los literarios) es que es precisamente el tema lo que consigue relacionar elementos externos aparentemente desconectados. Veamos un ejemplo;

(6) María hacía punto

La Tierra gira alrededor del Sol

Van Dijk (1983:43) presenta (6) como ejemplo de un texto sin coherencia, porque en principio el que María haga punto no tendría que ver con el hecho de que la Tierra gire alrededor del Sol. Se trata, por tanto, para él, de un texto sin coherencia, porque los hechos mismos señalados estarían desconectados. Pues bien, es perfectamente posible incluir estas dos secuencias en un texto poético que cante, por ejemplo, el asombro ante lo ordinario, de modo que sería precisamente este tema lo que daría unidad a estas secuencias ( $\mathrm{y}$ a los hechos mismos). Así, el nuevo tetxo podría decir algo como:

(7) «Todo estaba bien,

Todo estaba en su sitio:

María hacía punto,

la Tierra gira alrededor del Sol.»

Aquí lo definitivo es que es precisamente el tema lo que pone en relación estos dos hechos aparentemente no conectados: la unidad sólo se percibe con relación al tema, con relación a un principio interno al texto. Naturalmente, hemos alterado el texto inicial al pasar de (6) a (7), pero esto no importa, si tenemos en cuenta que lo que se trata de mostrar es que es precisamente el tema el que pone la relación entre las cosas, porque sin relación a este tema se trataría de hechos desconectados.

\section{LA CONTRIBUCIÓN DEL CONOCIMIENTO DEL MUNDO}

6.1. Esto no significa, naturalmente, que el conocimiento del mundo no intervenga en el proceso de atribuir coherencia a un texto; significa que interviene en un orden y jerarquía inversos al que normalmente cabría pensar. En concreto: la referencia a las cosas, la designación, se percibe temporalmente antes que la captación del sentido global del texto; pero las referencias a lo real se reinterpretan posteriormente en función del sentido: es decir, lo que percibimos temporalmente antes es la referencia a las cosas, pero lo que captamos lógicamente antes es el sentido. Veamos un ejemplo de cómo funciona este proceso. Supongamos una novela de ciencia ficción en la que se nos diga lo siguiente: 
(8) «Miriam quedó asombrada ante aquel extraño ser. Se parecía algo a un marciano, pero tenía un horrible color lechoso, y debajo de la nariz un curioso agujero membranoso y móvil, y naturalmente, no era capaz de entender nuestra lengua marciana».

Inicialmente nos situamos en el lugar de un ser hermano, y desde este punto de vista vamos interpretando el texto, pensando que se trata de la descripción de un extraterrestre; sin embargo, al final -y sólo al final- nos damos cuenta de que para que el texto tenga sentido debemos interpretarlo justamente al revés: se trata de un hombre visto por un marciano. Esta segunda interpretación se realiza precisamente en función del sentido: sólo de ese modo el texto resulta coherente.

6.2. En consecuencia: la coherencia puede ser explicada más fácilmente partiendo del texto mismo, en este caso, por el procedimiento del tópico del discurso; lo externo contribuye a la coherencia, pero se subordina al sentido.

6.3. Hasta ahora sólo ha dado respuestas satisfactorias la interpretación por el tópico del discurso. Sin embargo, caben otras explicaciones que partan del texto mismo. El concepto de sentido puede ser muy útil a este respecto. En cambio, pensamos que deben abandonarse definitivamente los intentos de dar cuenta de la coherencia por factores exteriores al propio texto, salvo que se conceda a éstos un valor tan sólo instrumental.

\section{Referencias bibliográficas}

ABADI, A. 1986: «Studies on connexity and coherence in Israel», J. S. Petöfi, E. Sözer 1986, 369-97.

BELLERT, I. 1970: «On a Condition of the Coherence of Texts», Semiotica 2, 335-336.

BERNÁRDEZ, E. 1982: Introducción a la lingüística del texto, Madrid, Espasa. BERNÁRDEZ, E. ed. 1987: Lingüística del texto, Madrid, Arco Libros.

BOSCH, P. 1985: «Contrains coherence, comprehension. Reflections on anaphora», E. Sözer 1984, 299-319.

COMBETTES, B. 1986: «Coréférence et connexité thématique dans le discours», M. Charolles, J.S. Petöfi, E. Sözer eds., 101-24 .

CONTE, M-E. 1988: «Condizioni di coerenza: ricerche di linguistica testuale», Pavia, Faculta di Lettere e Filosofia.

CONTE, M-E. 1988 a: «Deissi testuale ed anafora», Conte 1988, 13-28 .

CONTE, M-E. 1988 b: «Coerenza testuale», Conte 1988, 29-44.

CONTE, M-E. 1988 c: «Coerenza, interpretazione, reinterpretazione», Conte 1988, 79-91.

COSERIU, E. 1962: «Determinación y entorno», Teoría del lenguaje y lingüística 
general, Madrid, Gredos, 282-323.

COSERIU, E. 1978: «Lógica del lenguaje y lógica de la gramática», Gramática, semántica, universales, Madrid, Gredos, 15-49.

COSERIU, E. 1981 a: Textlinguistik. Eine Einführung, Tübingen, Narr.

COSERIU, E. 1981 b: «La socio- y la etnolingüística: sus fundamentos y sus tareas», Anuario de Letras 19, Mexico, 5-30.

COSERIU, E. 1985: «Linguistic Competence: What is it Really?», The Modern Lenguage Review 80,pp. XXV-XXXV.

CHAROLLES, M. 1978: «Introduction aux problemes de la coherence des textes», Langue Française 38, 7-41.

CHAROLLES, M. 1983: "Towards a heuristic approach to the textcoherence problems», F. Neubauer ed., 1-16.

CHAROLLES, M. 1985: «Text connexity, text coherence and text interpretation processing» E. Sòzer ed. 1985, 1-15.

CHAROLLES, M. 1986: «Le probléme de la coherence dans les études françoises sur le discours durant la periode 1965-1975», en M. Charolles, J. S. Petòfi y E. Sòzer eds 1986 3-60.

CHAROLLES, M.; PETÖFI, J. S. ; SÖZER, E. eds. 1986: Resears in Text Connexity and Text Coherence, Hamburg, Buske (Papers in Textlinguistics, 52, 1).

CHERCHI, L. 1978: «L' ellipse comme facteur de cohérence», Langue Fraņ̧aise 38, 118-128.

DIJK, T.A. van 1983, La Ciencia del texto, Barcelona, Paidós.

DUBOIS, J. y SUMP, J. 1970: «Un modèle d'enseignement du français: analyse linguistique des raports d'agregation et du CAPES», Langue Française $5,27-45$.

FILLOL, F. y MOUCHON, J. 1978: «Aproche des notions de cohérence et de cohésion sur un corpus oral», Langue Française, 38, 87-100.

GIORA, R. 1985: «What's a coherente text?», E. Sözer ed. 1985, 16-35.

HARRIS, Z.S. 1952: «Discourse Analysis», Language, 28, 1-30.

HATAKEYAMA, J; J. S. PETÖFI; E. SÖZER 1985: «Text, connexity, cohesion, coherence», E. Sözer ed. 1985, 36-105.

HOBBS, Jerry R. 1983: «Why is discourse coherent», F. Neubauer ed., 29-70.

LAPARRA, M. 1982: «Sélection thématique et cohérence du discours à l'oral», Le Français Moderne 50, 208-236.

LECOMTE, A. y J-M MARANDIN 1986: «Analyse du discourse et morphologie discursive», M. Charolles, J.S. Petöfi, E. Sözer eds., 61-100.

LORANT, B. 1985: «Conscious tradition, unconcious construction or subconscious metaphores? Certain levels of text cohesion and coherence», E. Sözer ed. 1985, 381-413.

LUNDQUIST, L. 1980: «La cohérence textuelle: syntaxe, sémantique, pragmatique», Copenhague, A. Busck.

LUNDQUIST, L. 1985: «Coherence: From structures to processes». E. Sözer ed. $1985,151-175$.

MALDIDIER, D. 1971 a: «Le discours politique de la guerre d'Algerie», 
Langages 23, 57-86.

MAINGUENEAU, D. 1976: Initiation aux méthodes de l'analyse du discourse, Paris, Hachette.

NEUBAUER, F. 1983: Coherence in Natural-Language Text, Hamburg, Buske (Papers in Textlinguistic, 38).

OZSEB, H. 1985: «Discoherent remarks on coherence», E. Sözer ed.1985, 567-594.

PECHEUX, M. 1969: Analyse automatique du discours, París, Dunod.

PETÖFI, J.S. 1975: Vers une théorie partielle du texte, Hamburg, Buske (Papers in Textlinguistic, 9).

PETÖFI, J.S. ed. 1979: Texte vs sentence. Basic Questions of Text Linguistics, Hamburg, Buske, 2 vols.

SGALL, P. 1983: «On one aspect of textual coherence», Neubauer ed, 135144.

SÖZER, E. ed. 1985: Text Connexity, Text Coherence. Aspects, Methods, Results, Hamburg, Buske.

VELDE, Roger G. van de 1985: «Inferences and coherence in text interpretation», E. Sözer ed. 1985, 261-98.

ZOLTAN, S. 1985: «The importance of text coherence for the global stylistic analysis», E. Sözer ed. 1985, 526-554. 